\title{
A homozygous duplication of the FGG exon 8-intron 8 junction causes congenital afibrinogenemia. Lessons learned from the study of a large consanguineous Turkish family
}

Haematologica 2022

Volume 107(5):1064-1071

\section{Correspondence:}

MARGUERITE NEERMAN-ARBEZ

Marguerite.Neerman-Arbez@unige.ch

Received: April 9, 2021.

Accepted: June 21, 2021.

Pre-published: July 1, 2021.

https://doi.org/10.3324/haematol.2021.278945

(C)2022 Ferrata Storti Foundation

Material published in Haematologica is covered by copyright. All rights are reserved to the Ferrata Storti Foundation. Use of published material is allowed under the following terms and conditions:

https://creativecommons.org/licenses/by-nc/4.0/legalcode. Copies of published material are allowed for personal or internal use. Sharing published material for non-commercial purposes is subject to the following conditions:

https://creativecommons.org/licenses/by-nc/4.0/legalcode, sect. 3. Reproducing and sharing published material for commercial purposes is not allowed without permission in writing from the publisher.

\section{Michel Guipponi, ${ }^{1,2}$ Frédéric Masclaux,${ }^{1}$ Frédérique Sloan-Béna, ${ }^{1}$ Corinne Di Sanza, ${ }^{2}$ Namik Özbek, ${ }^{3}$ Flora Peyvandi, ${ }^{4,5}$ Marzia Menegatti, ${ }^{4}$ Alessandro Casini, ${ }^{6}$ Baris Malbora ${ }^{7}$ and Marguerite Neerman-Arbez ${ }^{2}$}

${ }^{1}$ Medical Genetics Service, University Hospitals of Geneva, Geneva, Switzerland; ${ }^{2}$ Department of Genetic Medicine and Development, Faculty of Medicine, University of Geneva, Geneva, Switzerland; ${ }^{3}$ Department of Pediatric Hematology, Ankara City Hospital, Ankara, Turkey; ' Fondazione IRCCS Ca' Granda Ospedale Maggiore Policlinico, Angelo Bianchi Bonomi Hemophilia and Thrombosis Center and Fondazione Luigi Villa, Milan, Italy; ${ }^{5}$ Università degli Studi di Milano, Department of Pathophysiology and Transplantation, Milan, Italy; ${ }^{6}$ Division of Angiology and Hemostasis, University Hospitals of Geneva, Geneva, Switzerland and ${ }^{7}$ Department of Pediatric Hematology and Oncology, Istanbul Yeni Yuzyil University, Istanbul, Turkey

\section{ABSTRACT}

Q ongenital afibrinogenemia is the most severe congenital fibrinogen disorder, characterized by undetectable fibrinogen in circulation. Causative mutations can be divided into two main classes: null mutations with no protein production at all and missense mutations producing abnormal protein chains that are retained inside the cell. The vast majority of cases are due to single base pair mutations or small insertions or deletions in the coding regions or intron-exon junctions of $F G B$, $F G A$ and FGG. Only a few large rearrangements have been described, all deletions involving FGA. Here we report the characterization of a $403 \mathrm{bp}$ duplication of the FGG exon 8-intron 8 junction accounting for congenital afibrinogenemia in a large consanguineous family from Turkey. This mutation, which had escaped detection by Sanger sequencing of short polymerase chain reaction (PCR) amplicons of coding sequences and splice sites, was identified by studying multiple alignments of reads obtained from whole exome sequencing of a heterozygous individual followed by PCR amplification and sequencing of a larger portion of $F G G$. Because the mutation duplicates the donor splice site of intron 8, we predicted that the impact of the mutation would be on FGG transcript splicing. Analysis of mRNA produced by cells transiently transfected with normal or mutant minigene constructs showed that the duplication causes production of several aberrant FGG transcripts generating premature truncating codons.

\section{Introduction}

The ultimate goal of the coagulation cascade is the controlled conversion by thrombin of fibrinogen into fibrin which forms a polymer to give stability, strength and adhesive surfaces to growing blood clots containing platelets and red blood cells. Human fibrinogen ${ }^{1,2}$ is produced in the liver from three homologous polypeptide chains, $B \beta, A \alpha$ and $\gamma$ encoded by the fibrinogen gene cluster $F G B$, $F G A$ and $F G G$, on human chromosome 4. Two copies of each polypeptide chain assemble to form a hexamer $(A \alpha B \beta \gamma)$, held together by disulphide bonds. Alternative spliced transcripts are produced for $F G A$ and $F G G$, these are $\mathrm{A} \alpha \mathrm{E}$ and $\gamma^{\prime}$ respectively. While $\mathrm{A} \alpha \mathrm{E}$ chains are present in only $1-2 \%$ of circulating fibrinogen, $\gamma^{\prime}$ chains are present in $8-15 \%$ of circulating fibrinogen, in het- 
erodimeric or homodimeric form. Normal plasma fibrinogen levels vary between 2 and $4 \mathrm{~g} \mathrm{~L}^{-1}$. Variation in fibrinogen levels is a complex trait, influenced by both the environment and genotype.

Inherited disorders of fibrinogen include Type I disorders (afibrinogenemia and hypofibrinogenemia) which affect the quantity of fibrinogen in circulation and Type II disorders (dysfibrinogenemia and hypodysfibrinogene$\mathrm{mia}$ ) which affect the quality of circulating fibrinogen. . $^{3,4}$ Congenital afibrinogenemia is the most severe disorder, characterized by undetectable fibrinogen in circulation., ${ }^{5,6}$

While the first dysfibrinogenemia mutation was identified as early as $1968^{7}$ the molecular basis of afibrinogenemia was elucidated much later. ${ }^{8}$ This disorder is characterized by autosomal recessive inheritance and the complete absence of fibrinogen in plasma. In populations where consanguineous marriages are common, the prevalence of afibrinogenemia is increased. ${ }^{9}$

We identified the first causative mutation for congenital afibrinogenemia, a large, recurrent deletion in FGA in $1999^{\circ}$ identified in homozygosity in four members of a Swiss family. Since then, the underlying molecular pathophysiology of numerous causative mutations leading to fibrinogen deficiency has been determined by our group and many others (reviewed in $\left.{ }^{4}\right)^{10-12}$

Causative mutations can be divided into two main classes: null mutations with no protein production at all and missense mutations producing abnormal protein chains that are retained inside the cell. The vast majority of cases are due to single base pair mutations or small insertions of deletions in the coding regions or intron-exon junctions of $F G B, F G A$ and $F G G$. These can easily be identified by polymerase chain reaction (PCR) amplification followed by Sanger sequencing or by next-generation sequencing in particular whole exome sequencing (WES). Only a few large rearrangements have been described. In addition to the recurrent deletion we identified with breakpoints in FGA intron 1 and the FGA-FGB intergenic region, while three other large deletions in the fibrinogen gene cluster have been reported by others, all involving part of the FGA gene. These are a deletion of $1.2 \mathrm{~kb}$ eliminating the entire $F G A$ exon 4 in a Japanese patient; ${ }^{13}$ a deletion of $15 \mathrm{~kb}$, with breakpoints situated in $F G A$ intron 4 and in the $F G A-F G B$ intergenic region in a Thai patient: ${ }^{14}$ and a 4.1-kb deletion encompassing FGA exon 1 in an Italian patient. ${ }^{15}$ All patients were homozygous for the identified deletions except for the Thai patient, for whom complete maternal uniparental disomy was confirmed for the deleted chromosome $4 .^{14}$

Rearrangements of this type cannot be identified by simple PCR analysis of coding regions. Other techniques such as array comparative genomic hybridization (CGH) can be useful in some cases, however the resolution of commercial arrays limits the discovery of rearrangements i.e., deletions, duplications greater than $15 \mathrm{~kb}$. Consequently mutations less than $15 \mathrm{~kb}$ will escape detection using this technique in most diagnostic settings.

Here we report the characterization of a 403 bp duplication of the FGG exon 8-intron 8 junction accounting for congenital afibrinogenemia in a large consanguineous family from Turkey. This mutation, which had escaped detection by Sanger sequencing of short PCR amplicons of coding sequences and splice sites, was identified by studying multiple alignments of reads obtained from WES of a heterozygous individual followed by PCR amplification and sequencing of à larger portion of $F G G$. The mutation duplicates the donor splice site of intron 8 which leads to aberrant splicing of both the major $\gamma$ transcript and the minor $\gamma^{\prime}$ transcript.

\section{Methods}

\section{Patient samples}

This study was performed with Institutional Review Board approval and with written informed consent from all patients, in accordance with the Declaration of Helsinki. Platelet-poor plasma samples were obtained from citrated venous blood and analyzed as described in the Online Supplementary Appendix.

\section{Polymerase chain reaction and Sanger sequencing}

Genomic DNA was extracted from whole blood-EDTA according to standard protocols. PCR amplifications of all $F G B$, $F G A$ and $F G G$ coding regions and intron-exon junctions were performed as previously described. ${ }^{16}$ Standard primer sequences and PCR protocols are available on demand. Specific primer sequences for this study are available in the Online Supplementary Appendix. Sanger sequencing of purified PCR products was performed by Fasteris AG, Geneva, Switzerland.

\section{Array comparative genomic hybridization analysis}

The array CGH analysis was performed using Human Genome CGH Microarray Kit G3 1 M (Agilent Technologies, Palo Alto, USA) with $\sim 2.4 \mathrm{~kb}$ overall median probe spacing according to protocols provided by the manufacturers. Copy number variant analysis was done using the Agilent Genomic Workbench Software 7.0.4.0. and UCSC Genome Browser Human Genome GRCh37/hg19.

\section{Next-generation sequencing}

WES was performed at the Health 2030 Genome Center at Campus Biotech, Geneva using IDT Research Exome reagents. Read mapping and variant calling were performed using BWA 0.7.13, Picard 2.9.0, GATK HaplotypeCaller 3.7, aligned to the GRCh37/hg19 reference genome and annotated with Annovar 2017/07/17 and UCSC RefSeq (refGene) downloaded on $2018 / 08 / 10$

\section{Minigene constructs and transfections}

PCR products including intronic and exonic sequences from FGG intron 7 to $F G G$ exon 10 were amplified from the genomic DNA of one homozygous affected individual and one normal individual and cloned into the pcDNA3.1 V5His TOPO-TA eukaryotic expression vector (Invitrogen) to obtain mutant and normal minigenes. The presence of the $403 \mathrm{bp}$ duplicated fragment in the mutant clone was confirmed by Sanger sequencing. Transient transfections of HEK-293T cells $\left(10^{5}\right.$ cells/ condition) were performed in 6-well plates using Lipofectamine 2000 (Invitrogen) in OptiMEM (Gibco Invitrogen) and $2 \mu \mathrm{g}$ of normal or mutant construct. Two days post-transfection, cells were lyzed in Trizol for RNA extraction using the Turbo DNA free kit (Invitrogen). Reverse transcription and PCR amplification of cDNA for analysis of splicing variants are described in the Online Supplementary Appendix.

\section{Results}

The members of the large consanguineous family (Figure 1) all originate from a village in Turkey which 
keeps a religious faith different to the surrounding villages in the area. While the surrounding villages have a 'Sunni' faith, the village where the patients live has an Alavian-Bektashi faith. Since there are no marriages between these two religious groups, all marriages are between individuals from the same village. Interestingly, the original founders migrated from the Iranian province of Mazandaran, formerly known as Taberistan, during the 9 th and 10th centuries. Current inhabitants of Maznandar are also of Alavian-Bektashi faith.

Bleeding severity was assessed according to the score from the EN-RBD study. ${ }^{17}$ Patients were divided into clinical bleeding severity categories (asymptomatic and grade 1,2 , or 3 bleeding). Category 1 refers to provoked bleeding episodes, category 2 refers to spontaneous minor bleeding episodes (e.g., bruising), and category 3 refers to spontaneous major bleeding episodes (e.g., cerebral bleeds or hemarthrosis). ${ }^{17}$ Of the eight patients diagnosed with afibrinogenemia (Figure 1), four were available for genetic analysis. Fibrinogen measurements, both antigenic and coagulable, were performed for 41 additional family members (Table I).

Three patients available for study were male (patient ID: 1283, 1316, and 1317), and one was female (patient ID: 1314). All patients with afibrinogenemia have a severe clinical phenotype (grade 3) while most heterozygous patients with hypofibrinogenemia have a grade 0 (mean, 0.5). No patient received fibrinogen on prophylaxis. Two male patients with afibrinogenemia (1283 and
1317), for which no additional clinical information is available, have experienced a thrombotic event. This is not unusual; afibrinogenemia is associated with an increased thrombotic risk even in the absence of additional thrombotic risk factors, whether genetic or environmental. In a recent study of 204 afibrinogenemic patients, $37(18 \%)$ reported a thrombotic event. ${ }^{18}$

\section{Identification of a duplication of the FGG exon 8- intron 8 junction}

We aimed to identify the causative mutation by first screening three affected patients (1314, 1317 and 1283) and one heterozygous carrier (1288) by PCR amplification of all $F G B, F G A$ and FGG coding regions and intron-exon junctions followed by Sanger sequencing as previously described. ${ }^{16}$ This approach was unsuccessful, no causative mutation was identified. We then performed array-CGH analysis and identified 23 variants not listed in the database of Genomic Variants (http://dgv.tcag.ca/dgv/app/home). However none of these variants was a candidate for the afibrinogenemia phenotype so this approach was also unsuccessful.

Finally, as part of our ongoing research project determining the causative mutations and genetic modifiers of congenital fibrinogen disorders which uses WES followed by variant calling in a panel of selected genes including the fibrinogen genes, we included one heterozygous carrier, 1292, the mother of affected patient 1283, in the study. A detailed analysis of the reads suggested the pres-

A

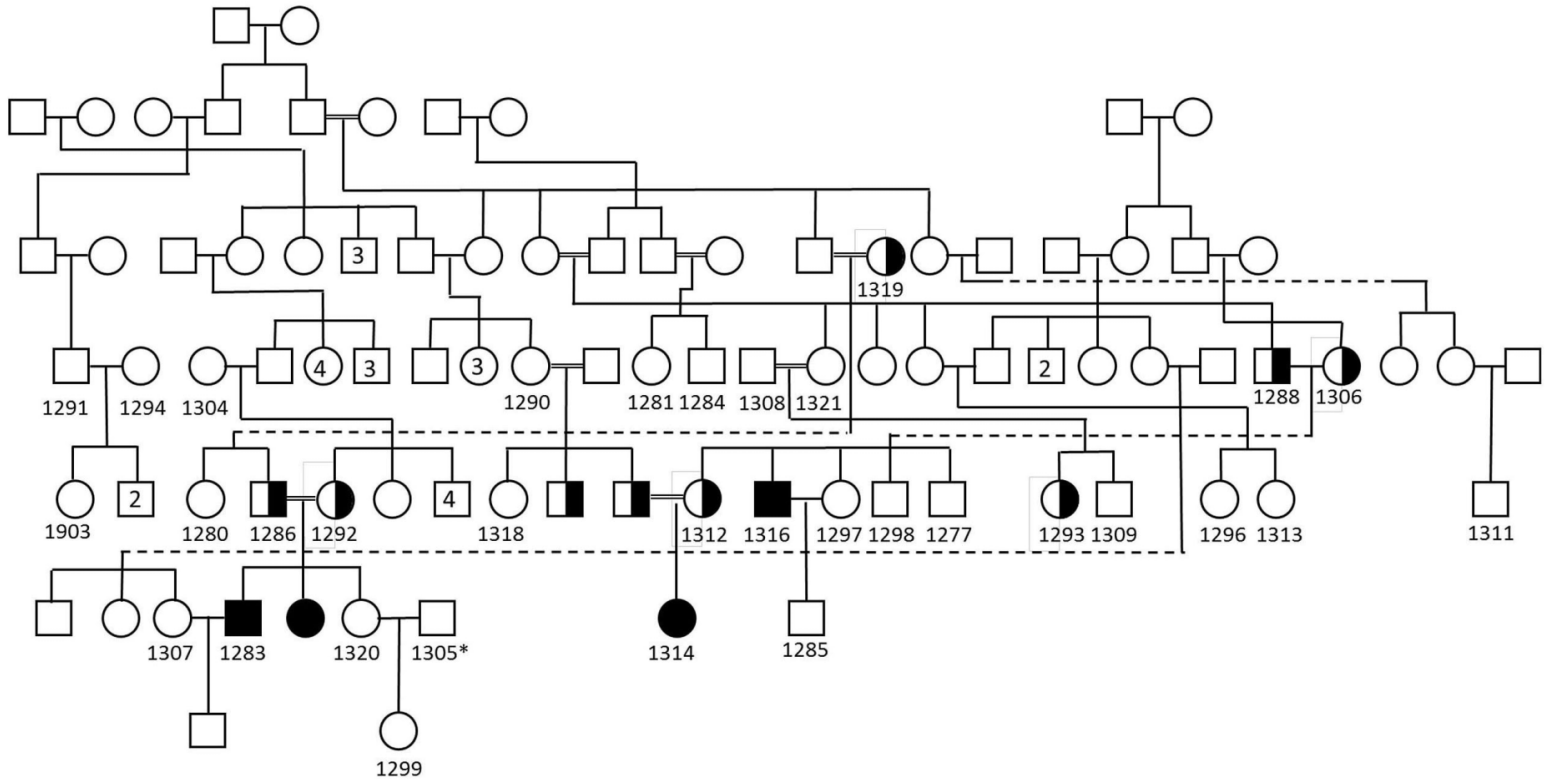

B

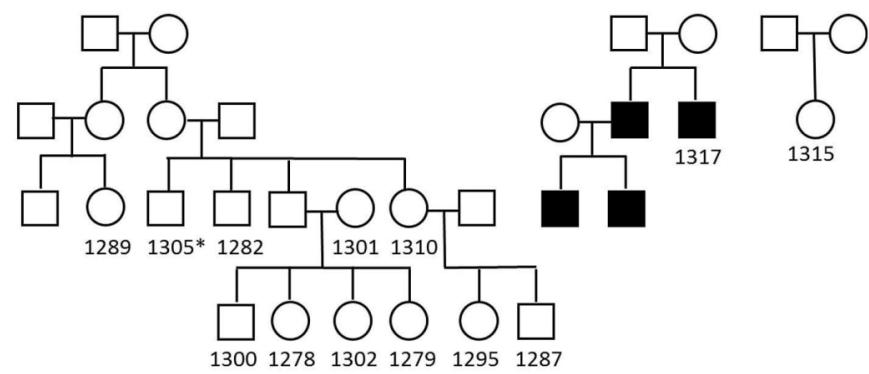

Figure 1. Family tree of the large consanguineous family. (A) Main family tree. (B) Other family members including relatives of individual 1305 (indicated with an asterisk). 
ence of an insertion of 36 bp in exon 8 of the FGG gene NM_021870.3,exon8,c.959_960insAATCCACCTGCTG CAAAATATCCAGTAGTTTGGCAT. This abnormality was present in only $14.2 \%$ of the reads (14/98) covering this position. The apparent localization of the insertion in FGG exon 8 was in contradiction with our previous results demonstrating the absence of a causative mutation in any fibrinogen gene coding sequence. BLAT analysis revealed that the $36 \mathrm{bp}$-long sequence was in fact in intron 8 of FGG suggesting the presence of a more complex local rearrangement. The visual inspection of the reads aligned to $F G G$ exon 8 further supported this hypothesis with the presence of hard clipped and outward-facing read pairs spanning far apart from that expected based on the library insert size (Figure 2). This configuration of the read pairs was highly suggestive of the presence of a heterozygous duplication that could not be fully characterized by our exon-centered analysis.

We therefore amplified by PCR a larger portion of $F G G$ from intron 7 to exon 10 which yielded a $4 \mathrm{~kb}$ product corresponding to the normal sequence and a $4.4 \mathrm{~kb}$ product for affected individuals. Heterozygous individuals showed both bands, as expected (Figure 3A). Sequencing of the larger band revealed the presence of a duplicated sequence of $403 \mathrm{bp}$ (out of 404, one base in a stretch of 4 intronic " $\mathrm{A}$ "s is missing) containing the last 169 bases from FGG exon 8, the donor splice site GT, and 232 additional bases of FGG intron 8 (Figure 3B). Genotypes for all family members are indicated in Table I. The presence of the duplication in homozygosity was associated with absence of fibrinogen in circulation and afibrinogenemia in all four affected individuals, while heterozygosity was associated with decreased fibrinogen levels (Table I).

\section{Duplication causes aberrant splicing of both FGG transcripts}

Because the mutation duplicates the donor splice site of intron 8 , we predicted that the impact of the mutation would be on $F G G$ transcript splicing. Interestingly, two FGG transcripts are normally produced which differ at the 3 'end: the major $\gamma$ chain mRNA has ten exons while in the minor $\gamma^{\prime}$ chain isoform intron 9 is not spliced out, substituting the four amino acids encoded by exon 10 with twenty $\gamma^{\prime} \mathrm{COOH}$-terminal residues. ${ }^{19.21}$ The presence of the duplication was thus anticipated to affect splicing of both isoforms. HEK-293T cells were transiently transfected with minigene constructs encompassing intron 7 to exon 10 with and without the duplication (Figure 4A). RNA produced were reverse transcribed to cDNA which were used in two different PCR reactions to amplify transcripts containing exon 10, present in the major $\gamma$ transcript, and transcripts containing the last bases of exon 9, retained in the minor $\gamma^{\prime}$ transcript but spliced out in the major $\gamma$ transcript. The results obtained for transcripts containing exon 10 (Figure 4B) showed one major product, indicated by an asterisk, for the normal minigene which was shown by sequencing to correspond to the correctly spliced mRNA containing exons 8, 9 and 10 encoding the major $\gamma$ chain. Sequencing of clones of individual PCR products showed that a transcript retaining intron 9 was also produced, thus encoding the minor $\gamma^{6}$ transcript even though exon 10 is present. For the mutant, the major product obtained, indicated by an asterisk, retained intron 8 with the duplication, resulting in a transcript with a frameshift and a premature truncating codon 13 codons downstream.

Cloning of the PCR products allowed the identification of additional minor aberrant transcripts resulting in frameshifts and premature truncating codons. One includ-

Table 1. Patient symptoms, fibrinogen measurements and genotypes.

\begin{tabular}{|c|c|c|c|c|c|}
\hline Patient ID & $\begin{array}{l}\text { Florinogen } \\
\text { clauss } \\
\text { (mg/dL) }\end{array}$ & $\begin{array}{l}\text { Florinogen } \\
\text { antigen } \\
\text { (mg/dL) }\end{array}$ & $\begin{array}{l}\text { Bleeding } \\
\text { score }\end{array}$ & Thrombosis & Genotype \\
\hline 1277 & 197 & 193 & 0 & & normal \\
\hline 1278 & 273 & 204 & 0 & & normal \\
\hline 1279 & 282 & 254 & 0 & & normal \\
\hline 1280 & 169 & 215 & 0 & & heterozygous \\
\hline 1281 & 214 & 218 & 0 & & normal \\
\hline 1282 & 231 & 206 & 0 & & normal \\
\hline 1283 & $<20$ & $<2$ & 3 & Yes & homozygous \\
\hline 1284 & 213 & 209 & 0 & & normal \\
\hline 1285 & 105 & 150 & 0 & & heterozygous \\
\hline 1286 & 152 & 177 & 0 & & heterozygous \\
\hline 1287 & 85 & 126 & 0 & & heterozygous \\
\hline 1288 & 152 & 170 & 1 & & heterozygous \\
\hline 1289 & 226 & 260 & 0 & & normal \\
\hline 1290 & 291 & 322 & 0 & & normal \\
\hline 1291 & 170 & 161 & 0 & & normal \\
\hline 1292 & 103 & 122 & 0 & & heterozygous \\
\hline 1293 & 115 & 110 & 2 & & heterozygous \\
\hline 1294 & 130 & 145 & 1 & & heterozygous \\
\hline 1295 & 273 & 320 & 0 & & heterozygous \\
\hline 1296 & 144 & 142 & 0 & & heterozygous \\
\hline 1297 & 186 & 175 & 0 & & normal \\
\hline 1298 & 317 & 334 & 0 & & normal \\
\hline 1299 & 246 & 267 & 0 & & normal \\
\hline 1300 & n.d. & n.d. & 0 & & normal \\
\hline 1301 & 272 & 260 & 0 & & normal \\
\hline 1302 & 334 & 342 & 0 & & normal \\
\hline 1303 & 90 & 86 & 2 & & heterozygous \\
\hline 1304 & 152 & 157 & 0 & & heterozygous \\
\hline 1305 & 269 & 267 & 0 & & normal \\
\hline 1306 & 108 & 132 & 1 & & heterozygous \\
\hline 1307 & 441 & 448 & 0 & & normal \\
\hline 1308 & 266 & 248 & 0 & & normal \\
\hline 1309 & 203 & 192 & 0 & & normal \\
\hline 1310 & 389 & 450 & 0 & & normal \\
\hline 1311 & 222 & 216 & 0 & & normal \\
\hline 1312 & 123 & 161 & 0 & & heterozygous \\
\hline 1313 & 149 & 187 & 0 & & heterozygous \\
\hline 1314 & $<20$ & $<2$ & 3 & & homzygous \\
\hline 1315 & 127 & 157 & 0 & & heterozygous \\
\hline 1316 & $<20$ & $<2$ & 3 & & homzygous \\
\hline 1317 & $<20$ & $<2$ & 3 & Yes & homzygous \\
\hline 1318 & 338 & 350 & 0 & & normal \\
\hline 1319 & 121 & 140 & 1 & & heterozygous \\
\hline 1320 & 179 & 220 & 1 & & normal \\
\hline 1321 & 165 & 171 & 0 & & heterozygous \\
\hline
\end{tabular}


A

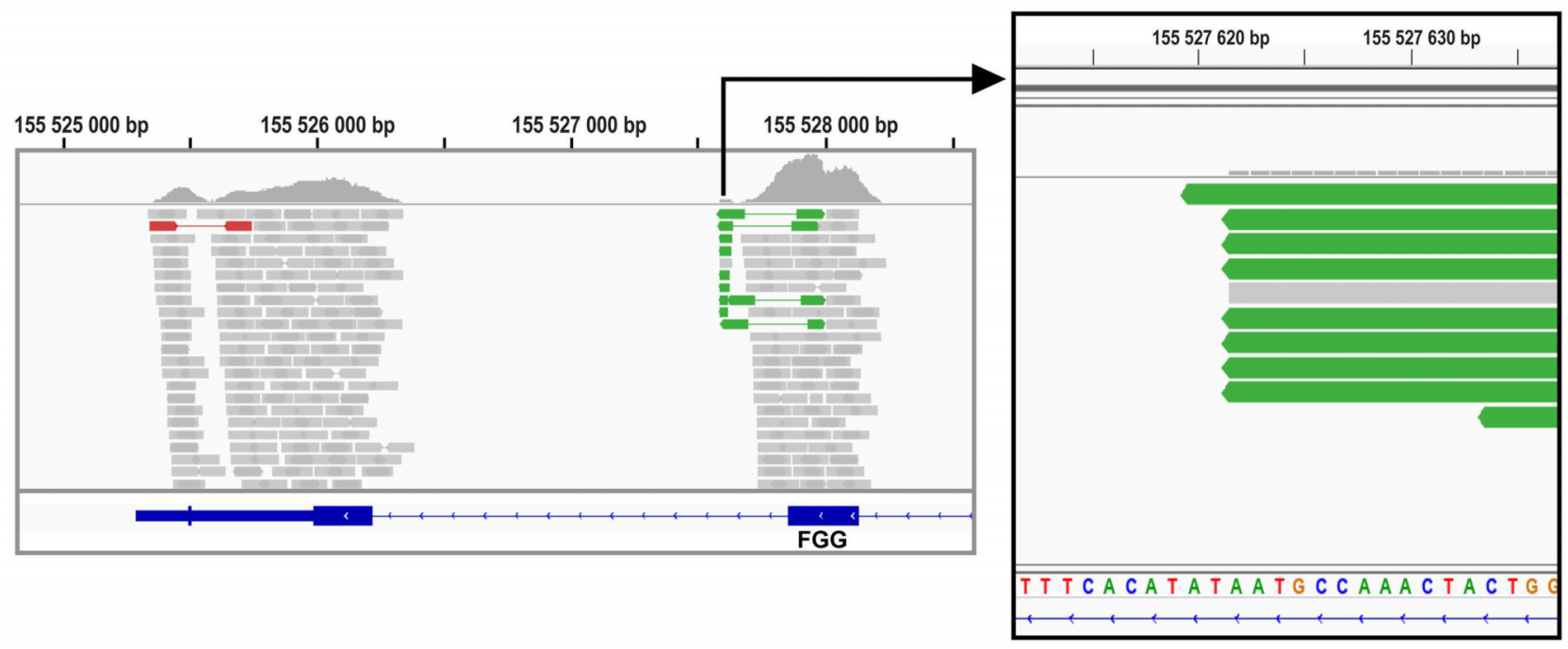

Figure 2. Whole exome sequencing read alignments. (A) IGV (Interactive Genome Viewer) screen capture of FGG gene showing reads between exon 7 and exon 10 (gene located on reverse strand, transcript NM_000509.5) aligned to the GRCh37/hg19 reference genome. Reads are colored by pair orientation as defined by standard IGV settings. Green color defines paired-end reads orientation inconsistencies which can delineate tandem duplication with respect to the reference genome. (B) Zoom on the reads that span the internal junction of the tandem duplication and only partially align to the reference genome (reads referred to as "hardclipped" at position 155'527'621).

A

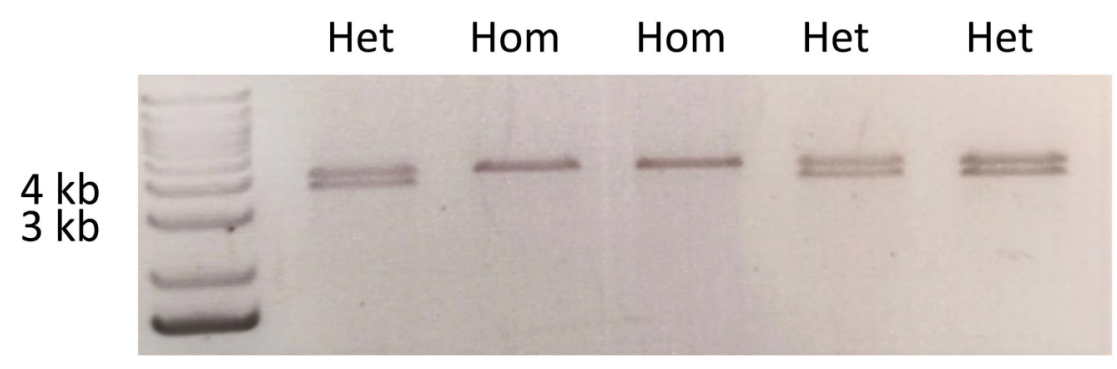

CATGTGATGGTTGTATTCCTTCTTCTCAGTACTGCAGACTATGCCATGTTCAAGgTGGGACCTGAAGCTGACAAGTACC GCCTAACATATGCCTACTTCGCTGGTGGGGATGCTGGAGATGCCTTTGATGGCTTTGATTTTGGCGATGATCCTAGTGAC AAGTTT'TTCACATCCCATAATGGCATGCAGTTCAGTACCTGGGACAATGACAATGATAAGTTTGAAGGCAACTGTGCTGA ACAGGATGGATCTGGTTGGTGGATGAACAAGTGTCACGCTGGCCATCTCAATGGAGTTTATTACCAAGGTATGTTTTCCT TTCTTAGATTCCAAGTTAATGTATAGTGTATACTATTTTCATAAAAAATAATAAATAGATATGAAGAAATGAAGAATAAT TTATAAAGATAGTAGGGATITIATCATGTTCTTTATITCAACTAAGTTCTTTGAAACTGGAAGTGGATAATACCAAGTTC ATGCCTAAAATTAGCCCTTCTAAAGAAATCCACCTGCTGCAAAATATCCAGTAGTTTGGCATTTTTGGCGATGATCCTAG TGACAAGTTTTTCACATCCCATAATGGCATGCAGTTCAGTACCTGGGACAATGACAATGATAAGTTTGAAGGCAACTGTG CTGAACAGGATGGATCTGGTTGGTGGATGAACAAGTGTCACGCTGGCCATCTCAATGGAGTTTATTACCAAGGTATGTTTT TCCTTTCTTAGATTCCAAGTTAATGTATAGTGTATACTATTTTCATAAAAAATAATAAATAGATATGAAGAAATGAAGAA TAATTTATAAAGATAGTAGGGATTTTATCATGTTCTITATITCAACTAAGTTCTITGAAACTGGAAGTGGATAATACCAA GTTCATGCCTAAAATTAGCCCTTCTAAAGAAATCCACCTGCTGCAAATATCCAGTAGTTTGGCATTATATGTGAAACTAT CACCATCATAGCTGG

Figure 3. Identification of a duplicated segment at the FGG exon 7-intron 8 junction. (A) PCR amplification of a portion of FGG from intron 7 to exon 10 yields a 4 $\mathrm{kb}$ product corresponding to the normal sequence and a $4.4 \mathrm{~kb}$ product for affected individuals. Heterozygous individuals show both bands. (B) Partial sequence of the $4.4 \mathrm{~kb}$ band. The acceptor "AG" site at the end of intron 7 and duplicated donor "GT" splice sites at the beginning of intron 8 are highlighted in yellow. Duplicated sequences are shown in blue. The duplicated sequence contains 403 bp out of 404 bp of the normal sequence, one base in a stretch of 4 intronic "A"s (shown in red) is missing. 


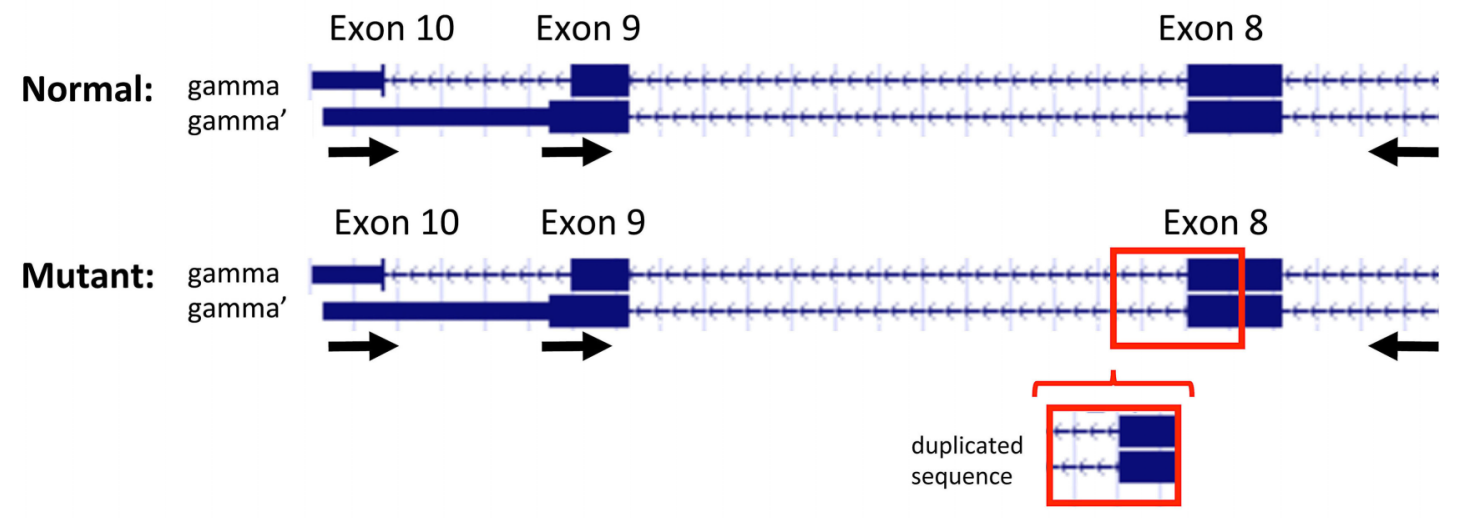

B

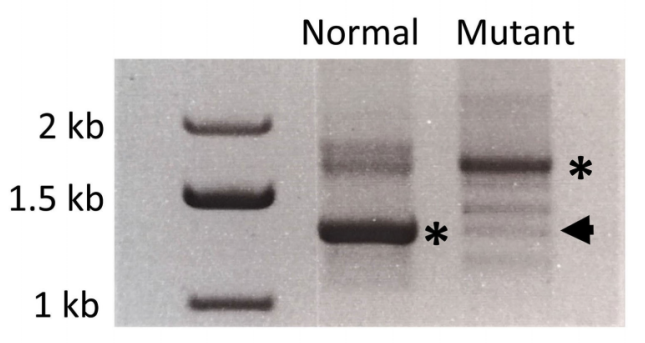

gamma
C

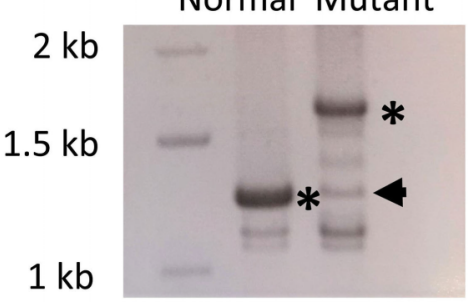

gamma'

Figure 4. Minigene constructs and reverse transcription polymerase chain reaction analysis of splicing variants. (A) Minigene constructs for the normal (top) and duplicated (bottom) FGG sequence between intron 7 and the 3'UTR of exon 10. HEK-293T cells transiently transfected with these constructs can produce transcripts for both the major $\gamma$ transcript, containing exon 10, and the minor $\gamma^{\prime}$ transcript, which does not splice out the 3' portion of exon 9. (B) cDNA obtained for transcripts containing exon 10. The major product for the normal minigene, indicated by an asterisk, corresponds to the correctly spliced mRNA containing exons 8,9 and 10 encoding the major $\gamma$ chain. For the mutant, the major product, indicated by an asterisk, retains intron 8 with the duplication, resulting in a transcript with a frameshift and a premature truncating codon. A correctly spliced transcript was also identified for the mutant minigene, but this is expected to be a rare event based on the intensity of the corresponding band indicated by an arrow. (C) cDNA obtained for transcripts containing the last bases of exon 9 retained in the minor $\gamma$ ' transcript. The major band for the normal minigene, shown with an asterisk, corresponds to the normal $\gamma^{\prime}$ transcript while the major transcript produced from the mutant minigene, retained intron 8 resulting in a frameshift as described above. Again, normal splicing is observed (indicated by an arrow) but at low levels.

ed part of the duplicated exonic segment due to utilisation of the normal donor splice site of intron 8 and a cryptic acceptor site situated in the duplicated sequence, followed by normal splicing of intron 9 using the duplicated donor site. Another transcript showed skipping of exon 9, joining together exon 8 and exon 10. The latter product was also identified in a clone of the normal minigene. Finally, a normal correctly spliced transcript was also identified for the mutant minigene, but this is expected to be a rare event based on the intensity of the corresponding band indicated by an arrow (Figure 4B). In order to have a complete picture of the different transcripts produced by alternative splicing of exon 9, we performed a second PCR amplification using a reverse primer localized on the last bases of exon 9 which are retained in the minor $\gamma^{\prime}$ transcript but spliced out in the $\gamma$ transcript. Again, one major product was identified for the normal construct and one for the mutant construct (Figure 4C). Sequencing confirmed production of the normal $\gamma^{\prime}$ transcript for cells transfected with the normal minigene while those transfected with the mutant minigene retained intron 8 resulting in a frameshift as described above.

\section{Discussion}

We describe here the identification of a homozygous duplication at the FGG exon 8-intron 8 junction account- ing for congenital afibrinogenemia in a large consanguineous family from Turkey. In principle identification of the mutation causing the complete deficiency of fibrinogen in the affected individuals, necessarily homozygous given the structure of the family pedigree, should be relatively straightforward for a laboratory equipped for standard genetic screening methods i.e., PCR amplification of the three fibrinogen encoding genes followed by Sanger sequencing. Indeed, we previously studied the molecular epidemiology of causative mutations for congenital fibrinogen disorders ${ }^{22}$ with the aim to design a cost- and time-effective screening strategy based on the genetic data from 266 unrelated patients genotyped in our laboratory. When we prospectively tested our strategy on 32 consecutive new probands we found that screening of FGA exons 2, 4, 5 and $F G G$ exon 8 combined with the search for the $11 \mathrm{~kb}$ deletion of FGA led to the identification of approximately $80 \%$ of mutated alleles, including 15 new mutations. ${ }^{22}$

In this case the size of the duplicated sequence (403 bp) was too large to be detected by standard PCR amplification approaches, which often involve amplifying single exons with only the immediate intronic sequences, and too small to be detected by array CGH. Identification of the mutation was only possible following a deep investigation of an aberrant sequence picked up by WES analysis but not initially confirmed by Sanger sequencing, an approach which is unlikely to be undertaken by routine diagnostic labora- 
tories. One lesson learned from this study is that amplifying larger overlapping portions of the genes of interest may allow the identification of similar mutations involving large insertions and duplications. In our case, amplifying for example FGG exons 7 to 10 , around $4,400 \mathrm{bp}$ in the normal sequence, and allowing sufficient elongation time to amplify the larger mutant band would have enabled the identification of the mutation sooner. Recent technology such as Nanopore DNA sequencing allowing sequencing of DNA fragments up to a few Mbp may be useful to identify such mutations, allowing for example the sequencing of the entire fibrinogen gene cluster with haplotype phasing of variant sites along the sequence.

The duplication is only one of five mutations involving rearrangements of more than $100 \mathrm{bp}$ of the fibrinogen genes, and the first identified in the FGG gene. As previously mentioned four deletions of several kilobases have been identified in FGA including the recurrent deletion we identified in a Swiss family. ${ }^{8,13-15}$ In addition, an inframe duplication of $117 \mathrm{bp}$ "Fibrinogen Champagne au Mont d'Or" leads to duplication of 39 amino acids within a repetitive sequence of 13 amino acids in the connector portion of the $\alpha \mathrm{C}$ domain ${ }^{23}$ a mutation predicted to cause an extension of the coiled coil. In $F G B$, while fibrinogen New York ${ }^{24}$ is described as a deletion of the amino acids encoded by $F G B$ exon 2 the mutation is not characterized at the DNA level and as mentioned by the authors is most probably due to a splice-site mutation leading to exon 2 skipping rather than deletion of exon 2 .

It is likely that many more mutations of this sort have remained elusive even for laboratories specialized in mutation identification. We hypothesized that the same duplication was likely to be found in other afibrinogenemic patients and their family members from the same geographical region or ethnic group. We therefore screened 10 unrelated patients from Turkey by PCR amplification and identified one additional afibrinogenemic patient who was homozygous for the same duplication. His parents, his grandmother and his two sisters were all heterozygous (data not shown). Enquiries into the geographical origin of this family revealed that they were originally from Kumbet village, Ortaköy, Aksaray, i.e., the same region as the first family.

While the nature of the mutation and the clear association with the phenotype i.e., complete fibrinogen deficiency with a homozygous genotype and partial fibrinogen deficiency with a heterozygous phenotype did not allow any reasonable doubt that we had identified the causative mutation we wished to identify the underlying molecular mechanism. Since the mutation duplicates the donor splice site of intron 8 we predicted that the mutation would impact $F G G$ transcript splicing, of both the major $\gamma$ transcript and the minor $\gamma^{\prime}$ transcript. Analysis of RNA produced by cells transiently transfected with normal or duplicated minigene constructs showed that in this model system the duplication causes production of several different aberrant transcripts of both isoforms leading to frameshifts and premature truncating codons.

Regarding the clinical significance of our findings, genotyping patients with fibrinogen disorders is now recommended in guidelines from the International Society of Thrombosis and Hemostasis. ${ }^{25}$ In quantitative fibrinogen disorders, the identification of the causative mutation(s) can help to distinguish between afibrinogenemia and severe hypofibrinogenemia. Providing an accurate diagnosis for these patients is important since in specific clinical settings such as pregnancy or surgery, patient management could be different.

In conclusion, we have identified a large duplication of several hundreds of basepairs at the FGG exon 8-intron 8 junction accounting for congenital afibrinogenemia in a large consanguineous Turkish family. The nature and size of the duplication can explain why this mutation was not identified using a standard PCR approach. It is highly likely that other patients with inherited quantitative fibrinogen disorders for whom no causative mutation has been identified harbor similar rearrangements.

\section{Disclosures}

$M G, F M, F S-B, M M, N O ̈, B M$ and $M N-A$ have no conflicts of interest to disclose; FP declares that she is a speaker at educational meetings and a member of advisory boards for Roche, Sanofi, SOBI and Takeda; AC declares grants and fees paid to his institution from Takeda and travel support from SOBI.

\section{Contributions}

$M G, F M, F S B$ and $C D S$ performed genetic experiments and interpreted the results; $N O, F P, M M, A C$ and $B M$ collected patient samples and clinical information and performed fibrinogen measurements; MNA directed the study and wrote the first draft of the manuscript. All authors contributed to writing and editing the final manuscript.

\section{Acknowledgements}

The authors thank Dr. Cédric Howald and Dr. Keith Harshman at the Health 2030 Genome Centre at Campus Biotech, Geneva for whole exome sequencing and initial processing of the data and Dr. Nermin Keni and Dr. Ekrem Unal for providing patient samples from the second Turkish family.

\section{Funding}

This study was funded by a grant from the Swiss National Science Foundation (grant \# 31003A_172864) to MNA.

\section{References}

1. Vilar R, Fish RJ, Casini A, Neerman-Arbez M. Fibrin(ogen) in human disease: both friend and foe. Haematologica. 2020;105(2): 284-296.

2. Pieters M, Wolberg AS. Fibrinogen and fibrin: an illustrated review. Res Pract Thromb Haemost. 2019;3(2):161-172.

3. Casini A, Brungs T, Lavenu-Bombled C, Vilar R, Neerman-Arbez M, de Moerloose P. Genetics, diagnosis and clinical features of congenital hypodysfibrinogenemia: a sys- tematic literature review and report of a novel mutation. J Thromb Haemost. 2017;15(5):876-888

4. Neerman-Arbez M, de Moerloose P, Casini A. Laboratory and genetic investigation of mutations accounting for congenital fibrinogen disorders. Semin Thromb Hemost. 2016;42(4):356-365.

5. Menegatti M, Peyvandi F. Treatment of rare factor deficiencies other than hemophilia. Blood. 2019;133(5):415-424.

6. Casini A, Neerman-Arbez M, de Moerloose P. Heterogeneity of congenital afibrinogene- mia, from epidemiology to clinical consequences and management. Blood Rev. 2020;26:100793.

7. Blomback M, Blomback B, Mammen EF, Prasad AS. Fibrinogen Detroit--a molecular defect in the $\mathrm{N}$-terminal disulphide knot of human fibrinogen? Nature. 1968;218(5137): 134-137.

8. Neerman-Arbez M, Honsberger A Antonarakis SE, Morris MA. Deletion of the fibrinogen [correction of fibrogen] alphachain gene (FGA) causes congenital afibrinogenemia. J Clin Invest. 1999;103(2):215-218. 
9. Peyvandi F, Mannucci PM. Rare coagulation disorders. Thromb Haemost. 1999;82(4): 1207-1214.

10. Casini A, de Moerloose P, Neerman-Arbez M. Clinical features and management of congenital fibrinogen deficiencies. Semin Thromb Hemost. 2016;42(4):366-374

11. Casini A, Neerman-Arbez M, Ariens RA, de Moerloose P. Dysfibrinogenemia: from molecular anomalies to clinical manifestations and management. J Thromb Haemost. 2015;13(6):909-919.

12. de Moerloose P, Casini A, Neerman-Arbez $M$. Congenital fibrinogen disorders: an update. Semin Thromb Hemost. 2013;39(6): 585-595.

13. Watanabe K, Shibuya A, Ishii E, et al. Identification of simultaneous mutation of fibrinogen alpha chain and protein $\mathrm{C}$ genes in a Japanese kindred. Br J Haematol. 2003;120(1):101-108

14. Spena S, Duga S, Asselta R, et al. Congenital afibrinogenaemia caused by uniparental isodisomy of chromosome 4 containing a novel $15-\mathrm{kb}$ deletion involving fibrinogen alpha-chain gene. Eur J Hum Genet. 2004;12(11):891-898.
15. Monaldini L, Asselta R, Duga $S$, et al. Mutational screening of six afibrinogenemic patients: identification and characterization of four novel molecular defects. Thromb Haemost. 2007;97(4):546-551

16. Neerman-Arbez M, de Moerloose P, Bridel $\mathrm{C}$, et al. Mutations in the fibrinogen aalpha gene account for the majority of cases of congenital afibrinogenemia. Blood. 2000;96 (1):149-152.

17. PeyvandiI F, Palla R, MenegattiI M, et al Coagulation factor activity and clinical bleeding severity in rare bleeding disorders: results from the European Network of Rare Bleeding Disorders. J Thromb Haemost. 2012;10(4):615-621.

18. Casini A, von Mackensen S, Santoro C, et al. Clinical phenotype, fibrinogen supplementation and health-related quality of life in patients with afibrinogenemia. Blood. 2021; 137(22):3127-3136

19. Mosesson MW, Siebenlist KR, Meh DA. The structure and biological features of fibrinogen and fibrin. Ann N Y Acad Sci. 2001:936:11-30.

20. Standeven KF, Ariens RA, Grant PJ. The molecular physiology and pathology of fib- rin structure/function. Blood Rev. 2005-19 (5):275-288

21. de Maat MP, Verschuur M. Fibrinogen heterogeneity: inherited and noninherited. Cur Opin Hematol. 2005;12(5):377-383.

22. Casini A, Blondon M, Tintillier V, et al. Mutational epidemiology of congenital fibrinogen disorders. Thromb Haemost. 2018;118(11):1867-1874

23. Hanss MM, Ffrench PO, Mornex JF, et al. Two novel fibrinogen variants found in patients with pulmonary embolism and their families. J Thromb Haemost. 2003;1(6): 1251-1257.

24. Liu CY, Koehn JA, Morgan FJ. Characterization of fibrinogen New York 1. A dysfunctional fibrinogen with a deletion of B beta(9-72) corresponding exactly to exon 2 of the gene. J Biol Chem. 1985;260 (7):4390-4396

25. Casini A, Undas A, Palla R, Thachil J, de Moerloose P, Subcommittee on Factor X, et al. Diagnosis and classification of congenital fibrinogen disorders: communication from the SSC of the ISTH. J Thromb Haemost. 2018;16(9):1887-1890 